\title{
Amenazas que enfrentan los monos congo (Alouatta palliata) en Costa Rica e iniciativas de conservación para el bienestar y una coexistencia sana con la especie
} Threats faced by howler monkeys (Alouatta palliata)
in Costa Rica and conservation initiatives for their well-being
and our healthy coexistence with the species

\author{
Gabriela Jones Román* \\ Cinthia Villalobos Suárez** \\ Rose Marie Menacho Odio***
}

DOl: https://doi.org/10.22458/rb.v32i1.3522

Recibido- Received: 09/03/2021 / Corregido- Revised: 12/03/2021 / Aceptado- Accepted: 25/03/2021

\section{RESUMEN}

El mono congo (Alouatta palliata) es una de las cuatro especies de primates de Costa Rica cuyo estatus de conservación la ubica como especie en peligro de extinción. La principal amenaza que enfrentan los monos congo es la pérdida del hábitat y la fragmentación del bosque situación que expone a sus poblaciones a problemas relacionados con el aislamiento, la pérdida de conectividad en el paisaje y un ambiente modificado por infraestructuras de origen antropogénico. Se expone la relevancia de atender la problemática que experimentan los monos congo en pro de su bienestar mediante el trabajo interinstitucional coordinado, la aplicación de la robusta legislación costarricense y el conocimiento ecológico existente dela especie.

Palabras clave: Allouatta paliatta; mono congo; pérdida de hábitat; fragmentación del bosque; electrocuciones; coexistencia.

\section{ABSTRACT}

The conservation status of the mantled howler monkey (Alouatta palliata), one of the four primate species in Costa Rica, is that of an endangered species. The main threats faced by howler monkeys are habitat loss and forest fragmentation, which expose monkey populations to problems related to isolation, loss of landscape connectivity, and an anthropogenically-modified environment. The following paper pinpoints the relevance of addressing the problems howler monkeys experience in order to ensure their well-being through coordinated inter-institutional work, the application of Costa Rica's robust legislation, and the existing ecological knowledge in the country about the species.

Key words: Allouatta paliatta; mantled howler monkey; habitat loss; forest fragmentation; electrocution; coexistence.

* Laboratorio de Ecología Urbana, Escuela de Ciencias Exactas y Naturales. Universidad Estatal a Distancia. gjones@uned.ac.cr ID: https://orcid.org/0000-0002-1363-4024

* Organización VerdeAgua. San José, Costa Rica. verdeaquacr@gmail.com ID: https://orcid.org/0000-0001-9240-5950

*** Programa de Manejo de Recursos Naturales, Escuela de Ciencias Exactas y Naturales. Universidad Estatal a Distancia. rmenacho@uned.ac.cr ID: https://orcid.org/0000-0002-2015-3376 


\section{Introducción}

El mono congo (Alouatta palliata) es una de las cuatro especies de primates neotropicales presentes en Costa Rica. Se encuentran naturalmente desde el sureste de México hasta el extremo norte de Perú. En Costa Rica, se hallan en todo el país tanto en bosques poco perturbados como también en hábitats altamente fragmentados. Sin embargo, se han extinguido en zonas muy urbanizadas que han perdido la mayor parte de su cobertura vegetal como es el caso del Gran Área Metropolitana (Francisco Durán comunicación personal, 2020).

La especie $A$. palliata se encuentra incluida en el Apéndice I de La Convención sobre el Comercio Internacional de Especies Amenazadas de Fauna y Flora Silvestre (CITES) y es catalogada como una especie vulnerable por la Unión Internacional para la Conservación de la Naturaleza (UICN). En Costa Rica, particularmente está catalogada como una especie en peligro de extinción, protegida y regulada por la Ley de

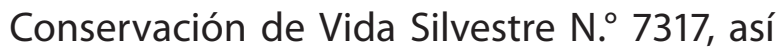
como por la Ley Orgánica del Ambiente N. 7554.

\section{Características alimentarias}

La dieta de los monos congo se describe como folívora-frugívora y se alimentan principalmente de brotes tiernos de hojas y frutos maduros, pero también consumen flores enteras, capullos y peciolos de una amplia variedad de especies de árboles tropicales y enredaderas (McKinney, 2018; Crockett, 1998). Se consideran una especie generalista en cuanto a su alimentación, condición que les permite sobrevivir ante los cambios en la abundancia de recursos alimentarios de hábitats con climas estacionales como el de la provincia de Guanacaste, donde pueden llegar a alimentarse de hasta 60 especies de plantas diferentes en un área determinada, característica que les permite ser muy flexibles en cuanto al alimento disponible a través del año (Stoner \& Timm, 2004). En Guanacaste, en los meses más secos, cuando más de $80 \%$ de los árboles del bosque tropical seco se encuentran sin hojas, los congos se alimentan de las hojas disponibles de especies siempreverdes como el guarumo (Cecropia peltata) y el espavel (Anacardium excelsum).

\section{Figura 1}

Los hábitos arborícolas de los monos congo

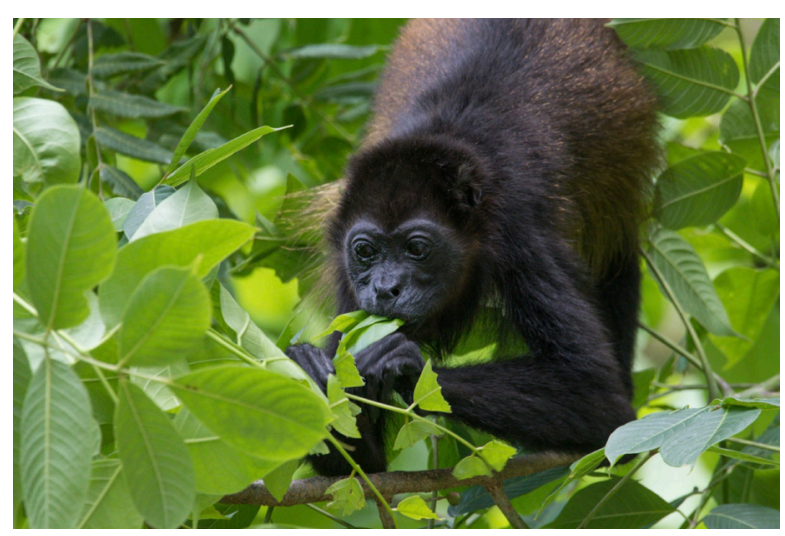

Nota. Su dieta, basada principalmente en hojas de diferentes especies de plantas, convierte a los bosques en elementos cruciales para la supervivencia de la especie. Fotografía de Luis José Venegas. Guanacaste, Costa Rica. 2020.

Algunas especies de plantas son sus preferidas, entre ellas, los frutos y hojas de diversas especies de Ficus spp. (McKinney, 2018; Milton, Armitage \& Sousa, 2019; Cristóbal-Azkarate y Arroyo-Rodríguez, 2007), el matapalo o chilamate (Ficus insípida, Milton, Armitage \& Sousa, 2019), los frutos y hojas de ojoche (Brosimun alicastrum, Milton, Armitage \& Sousa, 2019, Cristóbal-Azkarate y Arroyo-Rodríguez, 2007) 
así como los frutos, hojas y flores del espavel y el cenízaro (Samanea saman, McKinney, 2018).

Se cree que, en comparación con otras especies de monos, presentan una alta tolerancia a la destrucción del hábitat, en gran medida por su flexibilidad y capacidad de adaptación para variar sus estrategias de alimentación según el hábitat en que se encuentren (Clarke, Collins y Zucker, 2002) e inclusive la densidad de sus poblaciones se incrementa si aumenta la diversidad de alimentos en su dieta y el consumo de otros recursos no arbóreos como lianas, enredaderas, epífitas, arbustos, hierbas ypalmas (Cristóbal-Azkarate y Arroyo-Rodríguez, 2007).

\section{Principales problemáticas que afectan las poblaciones de monos congo}

A pesar de la mencionada plasticidad y capacidad de adaptación, los monos congo se enfrentan a varias amenazas que actualmente ponen en riesgo la salud y conservación de sus poblaciones a futuro. La pérdida y fragmentación del hábitat por el desarrollo urbanístico, el cambio de uso de suelo a pastizales o cultivos, además de la cacería, se han reconocido como las principales amenazas para la especie (Cortes-Ortíz et al., 2020).

La razón por la cual la pérdida y fragmentación del hábitat afectan el bienestar de las poblaciones de monos congo, es debido a que se desencadena una serie de efectos consecuentes que tienden a mermar o perjudicar a la especie. Por ejemplo, la fragmentación conlleva al aislamiento de las poblaciones, lo cual provoca un mayor riesgo de endogamia (cruce entre parientes) y consecuentemente a la pérdida de diversidad genética. Por otra parte, la disminución de conectividad en el paisaje limita el movimiento de los animales y el acceso a una mayor diversidad de fuentes de alimento (Blanco, 2019).

La pérdida del hábitat es la principal causa de extinción de especies. Una reducción del hábitat implica, para cualquier especie, una menor disponibilidad de recursos (refugio, alimento, pareja) y una menor calidad del hábitat disponible. En el caso particular de los congos se ha encontrado que los fragmentos de bosque ofrecen menos cantidad y calidad de recursos debido a la ausencia de árboles grandes, especialmente de algunas especies que son fundamentales en su dieta (ArroyoRodríguez \& Mandujano, 2006). La falta de algunas especies de árboles importantes puede provocar la disminución de las poblaciones de monos. Por ejemplo, en Panamá, se determinó que la disminución en densidad de dos especies de árboles de higuerón, cuyos frutos forman parte importante de la dieta de los congos, provocó una disminución en el tamaño de los grupos (Milton, Armitage, \& Sousa, 2019).

Otras consecuencias derivadas de la fragmentación del paisaje se relacionan con la discontinuidad de la cobertura forestal y las condiciones desfavorables de la matriz circundante a los fragmentos, situaciones que exponen a los congos a otros tipos de amenazas como el ataque por parte de perros, atropellos en carreteras y electrocuciones.

Los ataques de perros ocurren cuando los congos para desplazarse deben bajar al suelo por falta de conectividad entre las ramas; 0 bien, cuando son víctimas de una electrocución y quedan aturdidos o inmovilizados en el suelo. Se considera que el ataque por perros es la segunda causa de atención veterinaria 
para la especie, después de las electrocuciones, en el sector costero de Santa Cruz, Guanacaste (Gilberth Cavallini, comunicación personal, 2020).

Por otra parte, el atropello en carreteras es una causa antropogénica directa de mortalidad y no afecta únicamente a los monos congo. En un estudio realizado en la Ruta 32, carretera Braulio Carrillo entre San José y Limón, se identificaron al menos 25 especies diferentes de mamíferos muertos por atropello, entre ellas, los monos congo (Artavia et al., 2015).

Finalmente, la electrocución se perfila como la principal causa directa de muerte y lesiones en el caso de los monos congo que se reciben en dos de los principales centros de rescate de la provincia de Guanacaste (el Centro de Rescate y Santuario Las Pumas y el Refuge for Wildlife Nosara actualmente denominado International Animal Rescue), debido a accidentes ocurridos en las infraestructuras lineales de tipo eléctrico (Marta Cordero y Francisco Sánchez, 2020. Conversatorio "Experiencias de rescate de monos congo y sus principales protagonistas: desde el primer encuentro hasta su desenlace").

Para la Península de Nicoya se determinó que, entre enero de 2013 y enero de 2014, se electrocutaron en promedio dos animales por día, para un total de 774 individuos en ese año, se identificaron 97 accidentes de electrocución de mamíferos, 52 aves y 35 reptiles. Además, se observó que abril fue el mes con mayor número de electrocuciones, mientras diciembre y enero fueron los más bajos. Las localidades de Paquera, Filadelfia y Santa Cruz presentaron la mayor incidencia de averías por fauna, para un promedio de 115 individuos en ese período, seguidos por
Hojancha, Tamarindo, Nicoya y Hermosa con un promedio de 73 incidentes (Díaz 2014).

\section{Factores por considerar para una} prevención efectiva de incidentes de electrocución que involucre a las poblaciones de monos congo

En Costa Rica, los incidentes de electrocuciones de monos congos, a pesar de ser una problemática identificada, no se ha abordado de forma puntual espacial y temporalmente por lo que se desconoce la magnitud del problema a lo largo del tiempo y el impacto que podría estar teniendo en las poblaciones de monos congo,para más detalles sobre la problemática puede consultar los trabajos de Arauz (2002), Díaz (2014), Echandi (2018), Rodríguez et al. (2020).

Según datos recopilados en la Guía para la prevención y mitigación de la electrocución de la fauna silvestre por tendidos eléctricos en Costa Rica (2020), durante junio 2018 y junio 2019, se presentaron 947 casos de electrocuciones de primates no humanos. Sin embargo, la cifra representa únicamente eventos detectados y reportados. Aquellos incidentes que no generaron ninguna avería no fueron contabilizados y no todas las compañías electrificadoras llevan un registro de estos sucesos (Rodríguez et al., 2020). De lo anterior, se infiere que las cifras están subestimadas por un subregistro de información y porque no todo incidente genera una interrupción del servicio eléctrico que pueda ser cuantificado.

Tomando en consideración que algunos sectores costeros del Pacífico Norte están en franca expansión (Bahía de Papagayo, Bahía de Tamarindo, Playas del Coco, Nosara y Sámara) y que la cobertura arbórea se ha recuperado 
a nivel nacional y muy especialmente en áreas rurales de alto flujo y crecimiento turístico, es de esperar que la problemática de las electrocuciones esté en aumento y requiera de un análisis preventivo inmediato en aras de conservar una especie en peligro de extinción así como la imagen país que se desarrolla en armonía con la naturaleza.

Figura 2

Macho adulto junto a un transformador y sobre un entramado de cables semiaislados

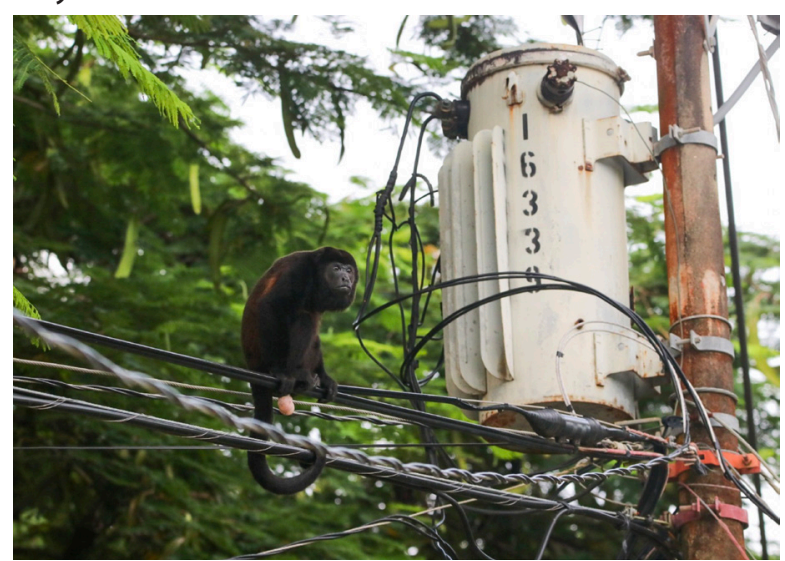

Nota. Fotografía de Luis José Venegas. Guanacaste,Costa Rica. 2020.

Los monos congo se diferencian del resto de las especies de primates del Nuevo Mundo, de hecho, de todos los mamíferos arborícolas del continente americano (excepto de los osos perezosos), por alimentarse primordialmente de hojas (Wainwright, 2007). Esta característica única de la especie hace que los monos congo naturalmente necesiten desplazarse en busca de alimento; además tanto las hembras como los machos juveniles tienden a abandonar su grupo natal para unirse a un nuevo grupo (Glander, 1992).

Bajo condiciones ambientales desfavorables, por ejemplo, sequías prolongadas, necesitan recorrer distancias mayores para suplir sus necesidades alimenticias. Lo anterior, en conjunto con la interrupción en la continuidad del bosque y la estacionalidad climática del Pacífico de Costa Rica, obliga a las tropas de congos a tener que desplazarse en una matriz de paisaje con diferentes usos del suelo, en los cuales se puedan presentar diversas amenazas que hacen de los congos una especie vulnerable a los efectos derivados de la fragmentación y la pérdida del hábitat.

Según Villalobos (2018), en algunas áreas de Costa Rica como en Liberia, Guanacaste, es posible encontrar grupos de individuos en parches muy pequeños de bosque $(1<\mathrm{Km})$ con escasa o nula conexión a áreas boscosas. Cuando las tropas de monos encuentran tendidos eléctricos como única alternativa de desplazamiento aéreo en sus rutas, muchas veces los utilizan para movilizarse, con el subsecuente riesgo de sufrir una electrocución. Estos eventos suelen ser frecuentes en zonas de Guanacaste como Playa Langosta y Tamarindo (Echandi, 2018).

Ahora bien, el abordaje preventivo no debe limitarse solo a considerar la biología de la especie y la gestión del paisaje, un aspecto medular de éxito de toda acción de conservación tendiente a la disminución de incidentes se relaciona con la coordinación entre entidades que velan por la conservación de la biodiversidad, el sector municipal que tiene una responsabilidad directa en el ordenamiento territorial, los permisos de construcción y los proveedores de servicios eléctricos que son los primeros que deben velar por la responsabilidad socio empresarial y la sostenibilidad ambiental de sus actividades.

En este sentido, el Informe Estado de la Nación (2020) alerta sobre un aumento de las amenazas que atentan sobre la calidad 
ecológica de la biodiversidad y la reducción en las capacidades financieras y humanas de los entes responsables de regular y fiscalizar la protección de la biodiversidad. Este último aspecto se está viendo materializado en los recientes recortes presupuestarios al Ministerio de Ambiente y Energía (MINAE), situación que debe ser seriamente abordada por las autoridades competentes en materia ambiental, como una señal urgente por atender para cumplir con el mandato de salvaguardar nuestra biodiversidad y de procurar un ambiente sano.

Una respuesta interinstitucional por parte del Estado en las acciones de prevención, fiscalización, atención de puntos críticos y de incidentes para disminuir el número de electrocuciones es una tarea que debe realizarse en apego a las responsabilidades establecidas por parte de los entes correspondientes en materia ambiental (MINAE-SINAC), de infraestructuras y desarrollo (Secretaría Técnica Nacional) y de regulación de servicios públicos (ARESEP) amparados en la extensa legislación y reglamentos existentes (Constitución Política de Costa Rica-artículo 50, Reglamento de la Ley de Conservación de la Vida Silvestre, decreto 40548-MINAE artículo 3 y 67, Ley 7317- artículo 6, Ley Forestal 7575 artículo 5, Ley de Biodiversidad 7878, entre otros).

Finalmente, en cuanto a las medidas técnicas clave para solventar la problemática en discusión se hallan: la poda preventiva y continua de las ramas cercanas a los tendidos eléctri$\cos y$ no eléctricos (tendidos telefónicos y de cable), el aislamiento de líneas eléctricas y transformadores en zonas de alto tránsito de fauna y la instalación de pasos aéreos que promuevan un desplazamiento efectivo y seguro de las tropas. En relación con estas medidas técnicas, se requiere de la participación activa de las comunidades y de los técnicos e investigadores en la identificación y selección de sitios prioritarios por atender.

\section{Algunas reflexiones para promover el bienestar de las poblaciones de monos congo e iniciativas de conservación que promuevan una} sana coexistencia con la especie

Los monos congo se perfilan como un potencial indicador del grado de conectividad en áreas fragmentadas (Hernández, 2015), debido a su presencia y permanencia en ambientes perturbados, a su capacidad de desplazamiento a través de diferentes hábitats en el paisaje y a su plasticidad en la utilización de recursos a partir de diversos usos del suelo. No obstante, en Costa Rica, se ha encontrado que la especie posee una baja variabilidad genética a nivel de sus poblaciones, situación que en ambientes fragmentados agrava su estatus de conservación (Rodríguez-Matamoros et al., 2012). Debido a estas particularidades de la biología de la especie y a que se ha observado que los fragmentos más pequeños y aislados en el paisaje tienen una menor calidad de hábitat (Arroyo-Rodríguez \& Mandujano, 2006) es necesario conservar remanentes de bosques grandes y potenciar el aumento de parches de mediano y pequeño tamaño (CristóbalAzkarate y Arroyo-Rodríguez, 2007).

Por otra parte, se debe promover que los fragmentos de bosques posean un mosaico heterogéneo de hábitats que brinden recursos alimentarios variados y agua disponible (Tinsley et al., 2020). También, se considera que la disponibilidad de árboles grandes y la 
presencia de frutos son dos elementos claves para la conservación de los congos en bosques fragmentados (Dunn et al., 2009).

Para la conservación de primates a largo plazo es importante hacer un esfuerzo para aumentar la permeabilidad de la matriz en el paisaje, conservar tantos remanentes de bosques como sea posible (de todo tamaño) y los bosques de galería (Oliveira et al., 2020). Los ambientes ribereños (vegetación asociada a los ríos y quebradas) deberían ser priorizados en la conservación de los monos congo porque la especie suele encontrarse asociada a este tipo de hábitat y además son ambientes que se establecen como zonas de protección en la Ley Forestal N. ${ }^{\circ} 7575$, artículo 33. En los ambientes ribereños se encuentra una mayor riqueza de especies de árboles e individuos de mayor tamaño, en los cuales se ha observado que los congos pasan una buena parte de su tiempo (Bolt et al., 2020).

En términos generales, se considera que todas las acciones tendientes a la regeneración y restauración del bosque deben ser contempladas como prioritarias en áreas en que se visualicen las condiciones de éxito a partir de las medidas que se implementen. El mejoramiento de la conectividad ecológica y la restauración de los ecosistemas a gran escala, mediados por la fauna nativa de un sitio, es una de las medidas que toma fuerza en la actualidad debido a su bajo costo y efectividad (Chazdon y Guariguata, 2016). Por tanto, se deben dedicar esfuerzos en la protección de grupos como los primates y las aves ya que se ha definido que la pérdida de especies en estos dos grupos influye negativamente en la capacidad de regeneración natural del bosque, dada la capacidad que tienen las especies citadas de desplazarse grandes distancias y así trasladar semillas a diferentes ambientes (Gardner et al. 2019).

En el caso específico de los monos congo, en una revisión realizada para el género Alouatta se mencionan tres estrategias clave que se deberían seguir para la conservación de la especie. La primera es incrementar el número, tamaño y la evaluación de las áreas protegidas para salvaguardar las poblaciones amenazadas; la segunda, expandir programas ambientales educativos locales para motivar actitudes y esquemas de manejo de la tierra que permitan la coexistencia de las personas y los monos; la tercera, translocar (reubicar) y reintroducir monos (Crockett 1998).

En relación con la primera medida, es importante mencionar que Costa Rica posee una extensa red de áreas protegidas que vendría a solventar, en alguna medida, la estrategia y a la vez estarían incentivando programas o proyectos que buscan mejorar la conectividad ecológica en el paisaje; por ejemplo, a través de la iniciativa nacional de corredores biológicos y de la promoción de la conectividad entre áreas protegidas mediante proyectos financiados con fondos del Primer Canje de Deuda por Naturaleza entre Estados Unidos y Costa Rica.

En cuanto a la segunda estrategia, se debe rescatar que el enfoque agropecuario del país, en consonancia con un modelo de desarrollo sostenible, podría facilitar un abordaje en esta línea hacia medidas de conservación de la especie, las cuales involucren de forma más activa a las comunidades y al sector productivo, especialmente en áreas donde aún se mantenga una matriz diversificada de actividades de bajo a moderado impacto ambiental en el paisaje. 
Es interesante rescatar que la utilización de recursos por parte de los congos en los agroecosistemas es bien tolerada por los agricultores en Mesoamérica y se considera que es parte del éxito de la especie en paisajes antropogénicos en sitios y épocas en que se presenta una alta abundancia de recursos alimentarios (McKinney, 2019). La regeneración natural de las tierras, que fueron en algún momento espacios dedicados a la agricultura y la ganadería, está convirtiendo un buen porcentaje de ese territorio en bosques en sucesión. Estos espacios con una cobertura vegetal en recuperación se constituyen en importantes reservorios de biodiversidad, que a la vez ofrecen diversos bienes y servicios ecosistémicos y pueden brindar diversas oportunidades de desarrollo que apoyen las economías y medios de vida rurales en Latinoamérica (Chazdon et al., 2020).

En Costa Rica, la provincia de Guanacaste es el referente de una deforestación postconquista que dedicó la mayor parte de sus tierras a la agricultura y a la ganadería y que actualmente está presentando una recuperación de su cobertura forestal en consonancia con un mayor desarrollo del sector turismo relacionado a los recursos naturales (PicadoUmaña y Cruz-Chaves, 2014). Es importante recalcar que en sitios donde la pérdida del hábitat es muy pronunciada y la problemática de electrocución es alta debería considerarse la translocación (traslado de un sitio a otro) de monos como una alternativa tendiente a la conservación y bienestar de las poblaciones locales.

Justamente, en torno a iniciativas de conservación de poblaciones locales de monos congo, destaca el trabajo que ha venido realizando la Asociación SalveMonos desde aproximadamente 17 años; inicialmente en la zona costera de Tamarindo y ahora con representación en otros sectores como Playa Hermosa y Playas del Coco. La Asociación se ha venido encargando de coordinar con la electrificadora la atención de zonas críticas, recibe los reportes de electrocuciones, recoge los monos heridos y coordina la atención veterinaria y el traslado a los centros de rescate cuando se amerita. También han apoyado algunas acciones de investigación, de reforestación y sensibilización con el apoyo de universidades y otras organizaciones locales. Las clínicas veterinarias y los centros de rescate de zonas aledañas. Mediante su labor de atención de animales heridos, rehabilitación y liberación de individuos han sido una pieza fundamental en todo un proceso de sensibilización y custodia hacia un proceso de empoderamiento local promotor de redes de integración público-privadas que conducen a una mejor gobernanza.

Finalmente, y en apoyo a estas iniciativas relacionadas con la problemática de conservación de los monos congo, desde agosto del 2020 se está realizando la campaña Protejamos los Monos Congo. La campaña surge como una iniciativa de la Comisión Bienestar Animal: todos somos individuos, convivimos en paz de la Dirección de Extensión de la Universidad Estatal a Distancia (UNED), en coordinación con el proyecto Monitoreo de fauna dispersora y polinizadora, desarrollo de capacidades comunales y restauración de hábitats ribereños para el mejoramiento de la conectividad ecológica entre el Parque Nacional Marino Las Baulas y el Parque Nacional Diriá, ejecutado por FUNDEPREDI Laboratorio de Ecología Urbana, financiado por el Primer Canje de Deuda por Naturaleza EE.UU.-CR y con la participación de la Cátedra de Ecología y Educación Ambiental de la 
Carrera de Manejo de Recursos Naturales, Escuela de Ciencias Exactas y Naturales de la Universidad Estatal a Distancia.

La campaña mencionada está orientada a generar y divulgar conocimiento, así como empatía hacia uno de los animales silvestres más comunes y visibles en los ecosistemas tropicales: los monos congo (Alouatta palliata). Se pretende difundir información biológica y ecológica relevante y sobre todo generar conciencia sobre la problemática que está experimentando esta especie de primate en Costa Rica a raíz de la pérdida de la conectividad en el paisaje, con el propósito de promover acciones para la conservación de estos animales, claves en la conectividad del paisaje y la salud del ecosistema tropical.

Dentro de la campaña Protejamos los Monos Congo-UNED se han trabajado tres objetivos: a) sensibilizar a la comunidad nacional respecto a la importancia y problemáticas que enfrenta la especie en Costa Rica, b) generar información científica y apoyar iniciativas para la conservación de la especie mediante ciencia ciudadana y c) desarrollar estrategias para el mejoramiento de la conectividad ecológica y la conservación de la especie en el paisaje.

Para lograr los objetivos descritos se han desarrollado conversatorios, infografías, afiches, y se ha apoyado la investigación a partir de ciencia ciudadana, así como acciones de manejo para la conservación de la especie. Para la divulgación de los contenidos de la Campaña y de otra información relevante en torno a la especie se han creado canales de comunicación como el blog El llamado del Mono Congo (https://llamadodelmonocongo.wordpress. com/), el grupo de Facebook Protejamos los Monos Congo y la página de Facebook Campaña Protejamos los Monos Congo-UNED.
En general, todas las actividades de la campaña pretenden llegar a un público de todas las edades, habitantes de localidades donde los monos congo formen parte de su paisaje natural, y de esta forma generar empatía y aprecio por esta especie de primate en riesgo de extinción.

\section{Referencias}

Arauz, E. (2002). Interacción de la Fauna Silvestre y la Red de Distribución Eléctrica: evaluación y soluciones de manejo en Costa Rica. Tesis de Maestría. Instituto Internacional en Conservación y Manejo de Vida Silvestre. Universidad Nacional. Heredia, Costa Rica.

Blanco, P. (2019). La realidad de los monos: I a antesala a la extinción. Retrieved from Universidad de Costa Rica Noticias Suplemento C+T: https://www.ucr.ac.cr/ noticias/2019/05/08/la-realidad-de-los-monos-laantesala-a-la-extincion.html

Bolt, L., Schreier, A., Voss, K., Sheehna, E. \& Barrickman, N. (2020). Down by the riverside: Riparian edge effects on three monkey species in a fragmented Costa Rican forest. Biotropica 52 (3): 541-553.

Chazdon, R.L \& Guariguata, M. R. (2016). Natural regeneration as a tool for large-scale forest restoration in the tropics: prospects and challenges. Biotropica 48: 716-730.

Chazdon, R., Lindenmayer, D., Guariguata, M.R., Crouzeilles, R., Rey, J.M. \& Lazos, E. (2020). Fostering natural forest regeneration on former agricultural land through economic and policy interventions. Environmental Research Letters 15: 1-16.

Clarke, M. R., Collins, D. A., \& Zucker, E. L. (2002). Responses to Deforestation in a Group of Mantled Howlers (Alouatta palliata) in Costa Rica. International Journal of Primatology, 365-381, Vol. 23.

Crockett, C. M. (1998). Conservation biology of the genus Alouatta. International Journal of Primatology 19(3):549-578.

Cortes-Ortíz, L., Canales Espinosa, D., Cornejo, F., GuzmanCaro, D. L., Méndez-Carvajal, P., Palacios, E.,de la Torre, S. (2020, February 3). Alouatta palliata. Retrieved from The IUCN Red List of Threatened Species: https://dx.doi.org/10.2305//UCN.UK.20202. rlts.t39960a17925090.en. 
Díaz, N. (2014). Impacto de la infraestructura eléctrica sobre la fauna silvestre de la Península de Nicoya, Costa Rica. Tesis de Maestría. Universidad Nacional, Instituto Internacional en Conservación y Manejo de Vida Silvestre. Heredia, Costa Rica.

Dunn, J. C., Cristóbal-Azkarate, J., \& Veà, J. (2009). Differences in diet and activity pattern between two groups of Alouatta palliata associated with the availability of big trees and fruit of top food taxa. American Journal of Primatology, 71, 654-662

Echandi, H. (2018). Electrocución de monos Congos (Alouatta palliata) en playa Tamarindo y Langosta, Guanacaste: una propuesta para la corrección y mitigación de estos accidentes. Tesis de Maestría. Programa de Estudios de Posgrado en Desarrollo Sostenible. Universidad de Costa Rica. 101 pp.

Gardner C J, Bicknell J E, Baldwin-Cantello W, Streubig M J y Davies Z G. (2019). Quantifying the impacts of defaunation on natural forest regeneration in a global meta-analysis. Nature Communications, 10, 4590.

Glander, K. (1992). Dispersal patterns in Costa Rican mantled howling monkeys. International Journal of Primatology 3:415-436. Hernández, J. E. (2015). Modelo para el establecimiento de corredores biológicos en áreas ambientalmente frágiles en el cantón Carrillo, Guanacaste, Costa Rica. Cartago, Costa Rica: Tésis licenciatura en ingeniería forestal, Instituto Tecnológico de Costa Rica.

Milton, K., Armitage, D. W., \& Sousa, W. P. (2019). Successional loss of two keys food tree species best explains decline in group size of Panamanian howler monkeys (Alouatta palliata). Biotropica, 1-15.

Oliveira, T., Macedo, R., Pinheiro, P., \& Rodrigues, F. (2020). Fragment shape and size, landscape permeability and fragmentation level as predictors of primate occupancy in a region of Brazilian Cerrado. Acta Scientiarum. Biological Sciences, 42: 2-15.

Picado-Umaña, W. y Cruz-Chaves, C. (2014). El bosque seco en llamas. Estructura agraria y ecología política del fuego en Costa Rica. Revista de Historia, 70, 109-142.

Protejamos los Monos Congo (5 de agosto 2020). Monos Congo e infraestructuras de desarrollo: intercambio de experiencias para una mejor convivencia. (Conversatorio). Facebook: https://www.facebook. com/rose.menacho/videos/10221247649474749
Protejamos los Monos Congo (26 de agosto 2020). Guía de prevención de electrocuciones. (Conversatorio). Facebook: $\quad$ https://www.facebook.com/rose. menacho/videos/10221399972682734

Protejamos los Monos Congo (30 de setiembre 2020). Experiencias de rescate de monos congo y sus principales protagonistas: desde el primer encuentro hasta su desenlace. (Conversatorio). Facebook: https://www.facebook.com/rose.menacho/ videos/10221641278515229

Rodríguez, K., Lara, L.R., Sánchez, A., Ramírez, D. \& S. Ramírez. (2020). Guía para la prevención y mitigación de la electrocución de la fauna silvestre por tendidos eléctricos en Costa Rica. Segunda Edición. Ministerio de Ambiente y Energía. San José, Costa Rica. 98p.

Rodríguez-Matamoros, J., Villalobos-Brenes, F \& GutierrezEspeleta, G. (2012). Viabilidad poblacional de Alouatta palliata (Primates: Atelidae) y Cebus capucinus (Primates: Cebidae) en el Refugio de Vida Silvestre Privado Nogal, Sarapiquí, Heredia, Costa Rica. Rev. Biol. Trop. 60 (2): 809-832.

Tinsley, E., Benítez, M., Fuentes, A., McLean, C., Norford, A., Ordoñez, J.C., Beehner, J., \& Bergman, T. (2020). High density of white-faced capuchins (Cebus capucinus) and habitat quality in the Taboga Forest of Costa Rica. American Journal of Primatology. 80 (2).

Villalobos, C. (2018). Determinación de la viabilidad de una población de monos congo Alouatta palliata (Atelidae) en un fragmento de bosque seco en Guanacaste, Costa Rica. Tesis de Licenciatura. Universidad Nacional, Costa Rica.

Wainwright, M. (2007). The Mammals of Costa Rica: A Natural History and Field Guide. Zona Tropical.

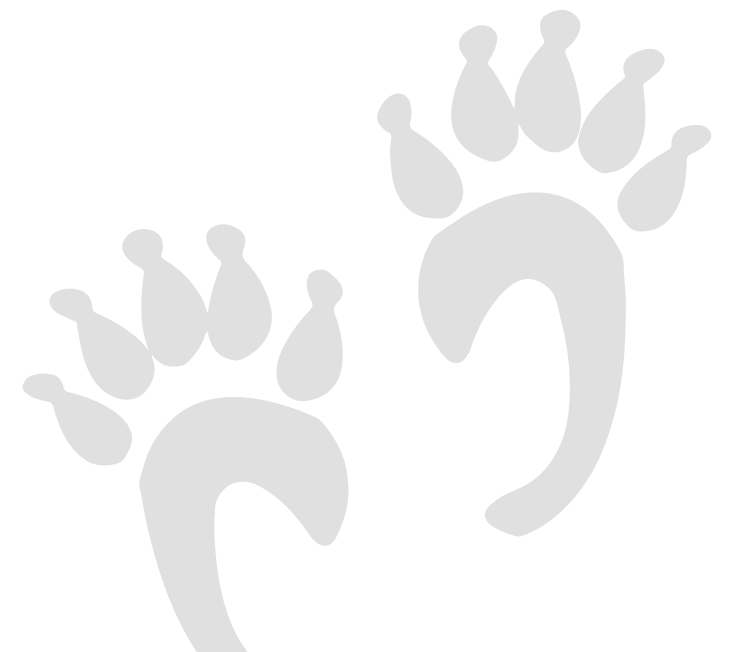

Biocenosis • Volumen 32/ Número 1/ junio 2021 